\title{
Fatores de risco associados ao desmame precoce e ao período de desmame em lactentes matriculados em creches
}

\author{
Risk factors associated to early weaning and to weaning period of infants enrolled in daycare centers
}

\author{
Marina Borelli Barbosa1 , Domingos Palma², Semíramis Martins A. Domene ${ }^{3}$, José Augusto A. C. Taddei ${ }^{4}$, Fábio Ancona Lopez ${ }^{5}$
}

\section{RESUMO}

Objetivo: Avaliar os fatores de risco no processo de desmame de lactentes matriculados em creches.

Métodos: Estudo analítico transversal com 56 crianças de nove a 18 meses de idade matriculadas em cinco creches de São Paulo. As mães das crinaças responderam ao questionário pré-testado que abordava questões demográficas, socioeconômicas, de gestação e nascimento, morbidade, aleitamento materno, alimentação complementar (quando/como foram introduzidos novos alimentos) e atividades da criança na creche. Os fatores associados ao desmame precoce e menor período de desmame (variável definida pela diferença entre tempo de aleitamento exclusivo e tempo de aleitamento total) foram avaliados em análises bivariadas. As variáveis com $p<0,10$ foram ajustadas em modelo conceitual hierárquico de regressão logística.

Resultados: $\mathrm{Na}$ análise multivariada, foram determinados os seguintes fatores de risco independentes para desmame precoce: renda familiar $\leq 3$ salários mínimos (OR 3,73; IC95\% 1,23-11,34); idade da mãe > 25 anos (OR 4,91; IC95\% 1,35-17,95); mãe morar sem companheiro (OR 6,42; IC95\% 1,28-32,20) e uso de chupeta antes dos 30 dias de vida (OR 8,75; IC95\% 1,90-40,23). Os fatores de risco para tempo insuficiente de desmame foram: frequentar creche pública (OR 3,20; IC95\% 0,77-14,29) e início tardio do pré-natal (OR 4,13; IC95\% 0,70-31,29).
Conclusões: Características relacionadas à família e à instituição creche são fatores de risco para o desmame de crianças institucionalizadas. A abordagem desses determinantes para definir ações de saúde e nutrição é estratégica para favorecer a prática do aleitamento e qualificar o papel da creche como promotora de saúde e nutrição infantil.

Palavras-chave: lactente; alimentação complementar; desmame; aleitamento materno.

\section{ABSTRACT}

Objective: To evaluate risk factors associated with weaning of infants enrolled in daycare centers.

Methods: Analytical cross-sectional study comprising 56 children aged nine to 18 months in five daycare centers of São Paulo. A pre-tested questionnaire was applied to their mother, including questions on demographics and socioeconomic issues, gestation and birth, illness, breastfeeding, complementary feeding (when and how new foods were introduced) and children activities in daycare centers. Factors associated with early weaning and short weaning period (defined as the difference between exclusive breastfeeding period and total breastfeeding period) were determined by bivariate analyses. Conceptual hierarchical multivariate analysis (logistic regression) was applied.

Results: In the multivariate analysis, the independent risk factors for early weaning were: family income $\leq 3$ minimum
Instituição: Departamento de Pediatria da Escola Paulista de Medicina da Universidade Federal de São Paulo (Unifesp-EPM), São Paulo, SP, Brasil ${ }^{1}$ Nutricionista; Mestre em Ciências da Saúde pelo Programa de Pósgraduação em Nutrição da Unifesp-EPM, São Paulo, SP, Brasil

${ }^{2}$ Pediatra; chefe da Disciplina de Nutrologia do Departamento de Pediatria da Unifesp-EPM, São Paulo, SP, Brasil

${ }^{3}$ Professora titular da Faculdade de Nutrição do Centro Ciências da Vida da Pontifícia Universidade Católica de Campinas, Campinas, SP, Brasil ${ }^{4}$ Professor-associado da Disciplina de Nutrologia do Departamento de Pediatria da Unifesp-EPM, São Paulo, SP, Brasil

${ }^{5}$ Professor titular da Disciplina de Nutrologia do Departamento de Pediatria da Unifesp-EPM, São Paulo, SP, Brasil
Autor para correspondência:

José Augusto A. C. Taddei

Disciplina de Nutrologia, Departamento de Pediatria, Unifesp-EPM

Rua Loefgreen, 1647 - Vila Clementino.

CEP 04040-032 - São Paulo/SP

E-mail: taddei.dped@epm.br

Fonte financiadora: Conselho Nacional de Desenvolvimento Científico e Tecnológico (CNPq), Edital - CT Agronegócio MCT/CNPq/MESA - 1/2003

- Projetos de pesquisa, desenvolvimento e inovação em Segurança Alimentar no Agronegócio, Processo n502930/2003-9 / Tema I: Segurança e Educação Alimentar.

Recebido em: 19/12/08

Aprovado em: 25/4/09 
wage (OR 3.73; 95\%CI 1.23-11.34); mother's age $>25$ years (OR 4.91; 95\%CI 1.35-17.95); mother living without a partner (OR 6.42; 95\% CI 1.28-32.20); use of pacifier before 30 days of life (OR 8.75; 95\% CI 1.90-40.23). Risk factors for insufficient weaning period were: attending public daycare centers (OR 3.20; 95\%CI 0.77-14.29) and late start of prenatal care (OR 4.13; 95\% CI 0.70-31.29).

Conclusions: Characteristics related to family and to daycare centers are risk factors for breastfeeding weaning among institutionalized infants. Such factors approach is strategic to define nutrition and health actions for favoring breastfeeding practice and to qualify daycare centers as infant health and nutrition promoters.

Key-words: infant; supplementary feeding; weaning; breastfeeding.

\section{Introdução}

O aleitamento materno e a alimentação adequada no primeiro ano de vida são os principais fatores determinantes do crescimento e desenvolvimento adequado e da condição de saúde da criança pequena ${ }^{(1,2)}$. Devido à alta velocidade de crescimento, a criança pequena é um dos grupos mais vulneráveis aos erros, deficiências e excessos alimentares, com consequências em seu estado nutricional ${ }^{(2,3)}$, como a desnutrição, a deficiência de micronutrientes ou o sobrepeso, devido à alimentação com excessos de açúcares, farináceos e gorduras ${ }^{(3)}$. Atualmente, há evidências de que a alimentação inadequada durante a primeira infância traz consequências importantes na condição de saúde a longo prazo, podendo ser um dos fatores que justifica o aparecimento das doenças crônicas na idade adulta ${ }^{(4,5)}$.

A Organização Mundial de Saúde (OMS), desde 2001, recomenda o aleitamento materno exclusivo até os primeiros seis meses de vida como medida de saúde pública e, após os seis meses, determina a introdução dos alimentos complementares com a manutenção do aleitamento materno até os dois anos de idade ou mais ${ }^{(6)}$. Esta recomendação também foi adotada em nosso país pelo Ministério da Saúde ${ }^{(7)}$.

O leite materno é alimento indispensável no início da vida, e inúmeras vantagens justificam a importância do aleitamento materno para a saúde da criança, da mãe e para a família nos seis primeiros meses de vida ${ }^{(7,8)}$. Trata-se de um alimento completo que fornece água, é isento de contaminação e perfeitamente adaptado ao metabolismo do bebê, rico em fatores de proteção contra diarreia e infecções, econômico, constituindo um adequado método de anticoncepção, além de promover o harmonioso vínculo entre mãe e filho ${ }^{(1,7-9)}$. Porém, diversos fatores podem influenciar de forma negativa ou positiva o sucesso do aleitamento materno, como características da mãe (idade, escolaridade, atitude da mãe em relação ao aleitamento, conhecimento e experiência anterior com amamentação), trabalho materno, condições socioeconômicas e de vida da família e orientações do profissional da saúde no pré-natal, no período pós-parto e puericultura ${ }^{(10)}$.

A complementação do leite materno com água ou líquidos não-nutritivos é desnecessária nos primeiros seis meses de vida, mesmo em dias secos e quentes. A introdução precoce desses líquidos não-nutritivos tem sido associada à menor duração do aleitamento materno, pois reduz o número de mamadas e diminui o volume de leite produzido ${ }^{(7)}$. Também a introdução da mamadeira e da chupeta pode confundir o reflexo de suç̧ão do recém-nascido e retardar o estabelecimento da lactação, pois os movimentos da língua e boca necessários para a sucção do peito são diferentes, além de diminuir a frequência da amamentação quando já estabelecida ${ }^{(7,9,11)}$.

Entretanto, a partir dos seis meses de vida, a alimentação complementar adequada é necessária para atender às necessidades nutricionais da idade. A introdução de novos alimentos é um processo complexo que envolve diversos fatores biológicos, culturais e socioeconômicos que determinam quando, como e porque iniciar o processo ${ }^{(2,7,9)}$. A alimentação complementar deve ser apropriada no tempo de introdução, adequada na forma de apresentação, quantidade e oferta, além de ser segura ${ }^{(1,2,4)}$.

O período de alimentação de transição é um dos fatores mais importantes na formação de hábitos alimentares saudáveis, pois o primeiro ano de vida é a época de formação da base destes hábitos ${ }^{(12)}$. A família é responsável pela formação do comportamento alimentar da criança por meio do aprendizado social ${ }^{(13)}$, representando os primeiros educadores nutricionais. Nesse contexto, a creche também exerce importante papel como parte integrante do processo de formação de hábitos alimentares de crianças pequenas, bem como incentivador à manutenção do aleitamento materno. Esta instituição atende, atualmente, a cerca de 290 mil lactentes pertencentes a famílias de baixa renda no município de São Paulo ${ }^{(14)}$ e é reconhecida como espaço de educação infantil, no qual ocorre o desenvolvimento desde os primeiros anos de vida, sendo a alimentação um dos aspectos a ser estimulado pelos educadores. Desta forma, família e creche podem juntas promover situações significativas de aprendizagem e convivência ${ }^{(15,16)}$.

$\mathrm{O}$ objetivo deste estudo foi identificar e quantificar os fatores de risco para desmame precoce e período de desmame 
de lactentes matriculados em creches do município de São Paulo e caracterizar a prática de alimentação complementar dessas crianças.

\section{Métodos}

Este estudo faz parte do "Projeto Crecheficiente: Ações de Segurança e Educação Alimentar em Creches Públicas e Filantrópicas do Município de São Paulo", que reuniu diversos profissionais com o objetivo de descrever a situação alimentar e as condições de saúde de crianças menores de dois anos que frequentam creches públicas e filantrópicas do município de São Paulo, para desenvolver mecanismos de supervisão e controle, visando a promover segurança alimentar e nutricional.

Trata-se de um estudo analítico transversal sobre a alimentação de transição de lactentes atendidos em cinco creches do município de São Paulo. A partir da listagem de creches vinculadas à Prefeitura Municipal de São Paulo, foram selecionadas as 31 instituições mais próximas à Instituição ou situadas em regiões de acesso facilitado pela linha de metrô, as quais foram consultadas por telefone quanto aos critérios de inclusão na pesquisa, como o número de crianças atendidas nos berçários, histórico de intervenções de universidades e aspectos da rotina das crianças. Foram então visitadas 13 creches para coleta de informações complementares para o desenvolvimento da pesquisa (estrutura de funcionamento, número de lactentes atendidos, número de educadores, recursos humanos disponíveis, condições de transporte dos pesquisadores e equipamentos). Nesta etapa, duas creches foram excluídas e as 11 restantes foram classificadas por painel de especialistas, de acordo com a metodologia descrita por Beghin ${ }^{(17)}$. Nesta metodologia, os critérios de seleção de maior interesse do estudo (maior número de lactentes e de educadores, ausência de intervenção de educação em saúde anterior, segurança na execução da pesquisa e atendimento a famílias de baixa renda) são listados de acordo com seu grau de importância, de forma a identificar, no final do processo, cinco instituições, sendo duas creches públicas e três filantrópicas as que melhor atenderam aos objetivos do estudo.

A coleta dos dados ocorreu entre os meses de agosto a outubro de 2004, período em que foram realizadas diversas atividades propostas pelo projeto, sendo que três delas fizeram parte deste estudo: entrevista com a mãe ou o responsável pela criança, entrevista com a direção de cada instituição sobre o esquema adotado para introduzir de alimentos por faixa etária e observação do preparo e da oferta desta alimen- tação. Antecedendo a coleta de dados, foi realizado o estudo piloto em uma creche filantrópica para teste da metodologia proposta e treinamento da equipe composta por pediatra, nutricionistas, cirurgiã-dentista e psicólogas.

O questionário para a entrevista com a mãe ou responsável continha questões abertas e fechadas que abordavam os seguintes aspectos: identificação da criança e da família, dados antropométricos da criança e da mãe, indicadores socioeconômicos, condições de gestação e nascimento, antecedentes mórbidos pessoais, aleitamento materno e alimentação complementar (quando e como foram introduzidos os novos alimentos), atividades da criança na creche e hábitos de higiene bucal. O questionário para a entrevista direcionada continha questões abertas sobre o esquema alimentar adotado pela instituição por faixa etária, o modo de preparo, a forma de oferta e as restrições que se seguiam por recomendação médica.

As variáveis contidas nos questionários, após avaliação da consistência de seu preenchimento, foram transcritas para um banco de dados desenvolvido no programa Epi-Info 2000, com dupla digitação e posterior validação.

A população estudada pelo Projeto Crecheficiente foi constituída pelo conjunto de crianças menores de dois anos regularmente matriculadas nas creches selecionadas, o que resultou em 218 indivíduos, sendo que cinco crianças com doenças agudas e uma que não estava presente durante as atividades de campo foram excluídas, totalizando 212 crianças. Para este estudo, foram selecionadas as crianças de ambos os gêneros com idade entre nove e 18 meses, com no mínimo cinco meses de matrícula na instituição e que já não estivessem mais sendo amamentadas. Este último critério de inclusão foi indispensável porque o tempo de aleitamento total subtraído do tempo de aleitamento exclusivo determinou a variável dependente "período de desmame". Dessa forma, após as restrições, para que se pudessem testar as hipóteses do estudo, a amostra ficou reduzida a 56 lactentes, sendo 28 do sexo masculino e 28 do feminino.

Para a análise estatística, foram escolhidas como variáveis dependentes: "tempo de aleitamento materno exclusivo" e "período de desmame", compreendido entre o fim do aleitamento exclusivo e o fim do aleitamento total, ou seja, o período em que se iniciou a introdução dos alimentos complementares. Utilizou-se como critério para categorização a mediana do tempo de aleitamento exclusivo, que foi de dois meses, e a mediana do período de desmame, que também foi de dois meses. Foram realizadas, inicialmente, análises descritivas univariadas, com o cálculo das proporções das variáveis categóricas por meio do qui-quadrado ou teste exato de Fisher. 
A associação entre o desmame precoce (aleitamento materno exclusivo $\leq 2$ meses) e o período insuficiente de desmame (período de desmame $\leq 2$ meses) com as variáveis independentes foram quantificadas a partir do cálculo das razões de chances (Odds Ratio) e intervalo de confiança de 95\% (IC95\%), avaliando-se sua significância estatística se $p<0,10$. A adoção de níveis de significância de 0.10 deve-se à natureza do estudo qualiquantitativo, que assume, dessa forma, uma probabilidade de erro alfa de $10 \%$.

A análise multivariada foi realizada ajustando-se o modelo de regressão logística para as variáveis dependentes de acordo com um modelo conceitual hierárquico ${ }^{(18)}$. No nível 1 , foram incluídas as variáveis consideradas distais e, nos níveis 2 e 3, as variáveis proximais. Selecionaram-se as variáveis com nível de significância menor que 0,10 para construir o modelo logístico e foi eleito o nível máximo de 0,05 para permanência no modelo final. A análise estatística foi realizada com o software Stata, versão 8.0.

O projeto foi aprovado pelo Comitê de Ética em Pesquisa da Universidade Federal de São Paulo.

\section{Resultados}

A duração média de aleitamento materno exclusivo na amostra foi $2,3 \pm 1,7$ meses e a mediana foi de dois meses. A duração média de aleitamento materno total destas crianças foi $5,1 \pm 3,7$ meses e a mediana, quatro meses. O período de desmame, que corresponde ao tempo entre o início da introdução dos novos alimentos e o tempo de aleitamento materno total, foi de 2,7 $\pm 3,2$ meses e a mediana, dois meses. Este resultado indicou que as crianças foram desmamadas pouco tempo após a introdução de um novo alimento.

Os motivos alegados pelas mães para introduzirem novos alimentos foram orientação médica (30,4\%), fatores ligados ao trabalho materno $(28,6 \%)$ e falta de conhecimento da mãe $(23,2 \%)$, entre outros $(17,8 \%)$.

O alimento inicialmente introduzido pela família foi o chá aos 2,8 $\pm 1,8$ meses. Em seguida, introduziu-se a água aos 3,4 2, 0 meses, o leite não materno aos 3,5 $\pm 2,2$ meses, o suco aos 4,5 $\pm 1,5$ meses, a papa de fruta aos $5,2 \pm 1,3$ meses e a papa salgada aos $5,4 \pm 1,3$ meses. A introdução dos alimentos na creche foi mais tardia do que a introdução feita pela família, como pode ser observado no Gráfico 1.

As análises referentes aos fatores de risco para as variáveis dependentes: desmame precoce (desmame precoce) e período de desmame (período insuficiente de desmame) estão apresentadas nas Tabelas 1 e 2 .

$\mathrm{Na}$ análise bivariada, foi possível identificar que a introdução da chupeta antes de 30 dias de vida aumenta o risco em 8,75 vezes para o desmame precoce $(p=0,005)$. Na

Idade (meses)

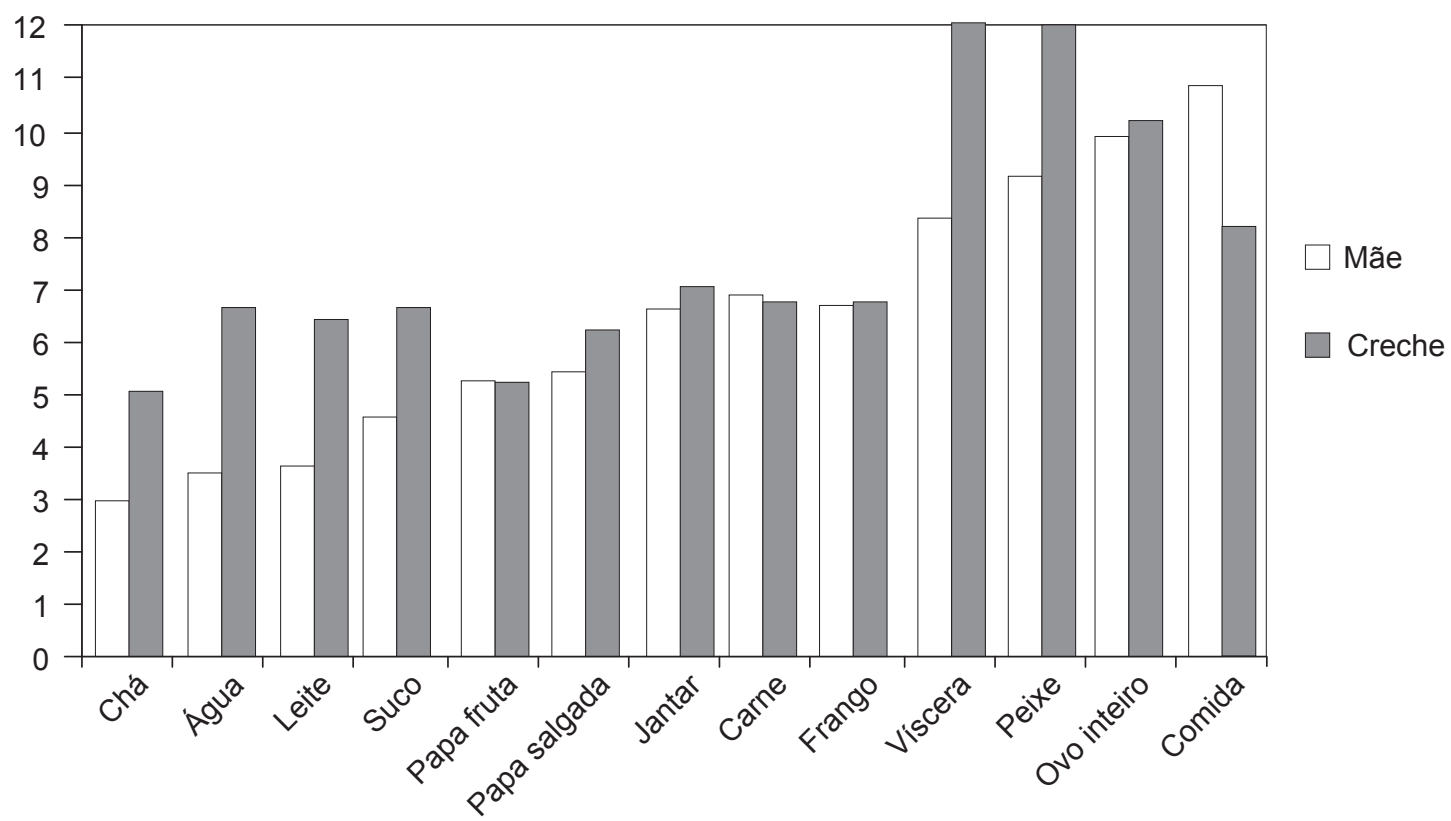

Gráfico 1 - Idade média da criança à introdução de alimentos pela mãe e pela creche. 
Tabela 1 - Prevalência de fatores de risco/proteção e Odds Ratios (OR) com respectivos intervalos de confiança de 95\% (IC95\%) para variáveis relacionadas ao desmame precoce de lactentes de nove a 18 meses

\begin{tabular}{|c|c|c|c|c|c|c|c|c|}
\hline & \multicolumn{6}{|c|}{ Desmame precoce } & \multirow{3}{*}{$\begin{array}{l}\text { OR Bruto } \\
\text { (IC 95\%) }\end{array}$} & \multirow{3}{*}{$\begin{array}{l}\text { Valor } \\
\text { de } p\end{array}$} \\
\hline & \multicolumn{3}{|c|}{$\leq 2$ meses } & \multicolumn{3}{|c|}{$>2$ meses } & & \\
\hline & $n_{\text {total }}$ & $\mathbf{n}$ & $\%$ & $\mathbf{n}_{\text {total }}$ & $\mathbf{n}$ & $\%$ & & \\
\hline \multicolumn{9}{|l|}{ Variáveis sociodemográficas e econômicas } \\
\hline Sexo masculino & 30 & 15 & 50,0 & 26 & 13 & 50,0 & $1,00(0,30-3,30)$ & 1 \\
\hline Creche pública & 30 & 13 & 43,3 & 26 & 7 & 26,9 & $2,08(0,58-7,58)$ & 0,201 \\
\hline Tempo de creche $<7$ meses (mediana) & 30 & 14 & 46,7 & 26 & 5 & 19,2 & $3,67(0,94-15,02)$ & 0,030 \\
\hline Mãe mora sem companheiro & 30 & 10 & 33,3 & 26 & 3 & 11,5 & $3,83(0,79-20,91)$ & 0,054 \\
\hline Primeiro filho & 30 & 10 & 33,3 & 26 & 8 & 30,8 & $1,13(0,31-4,07)$ & 0,838 \\
\hline Filho caçula & 30 & 20 & 66,7 & 26 & 18 & 69,2 & $0,89(0,25-3,20)$ & 0,838 \\
\hline Idade materna $>25$ anos & 30 & 22 & 73,3 & 26 & 12 & 46,1 & $3,20(1,05-9,81)$ & 0,038 \\
\hline Escolaridade materna $<4$ anos & 30 & 1 & 3,3 & 26 & 1 & 3,8 & $0,86(0,02-34,22)$ & 0,717 \\
\hline Moradia alugada ou cedida (não própria) & 30 & 24 & 80,0 & 26 & 20 & 76,9 & $1,20(0,28-5,18)$ & 0,779 \\
\hline Uso de banheiro coletivo & 30 & 8 & 26,7 & 26 & 3 & 11,5 & $2,79(0,55-15,64)$ & 0,155 \\
\hline Coleta de lixo $\leq 3$ vezes por semana & 30 & 18 & 60,0 & 26 & 13 & 50,0 & $1,50(0,45-5,03)$ & 0,453 \\
\hline Mãe desempregada & 30 & 7 & 23,3 & 26 & 6 & 23,1 & $1,01(0,25-4,22)$ & 0,982 \\
\hline Emprego da mãe não qualificado & 26 & 11 & 42,3 & 24 & 12 & 50,0 & $0,73(0,20-2,62)$ & 0,585 \\
\hline Chefe da família desempregado & 30 & 1 & 4,5 & 26 & 1 & 4,8 & $0,95(0,02-38,61)$ & 0,744 \\
\hline Emprego do chefe não qualificado & 21 & 6 & 28,6 & 20 & 2 & 10,0 & $3,60(0,52-31,07)$ & 0,134 \\
\hline Renda familiar $\leq 3$ salários mínimos & 30 & 21 & 70,0 & 26 & 10 & 38,5 & $3,73(1,07-13,50)$ & 0,017 \\
\hline Renda per capita $<1$ salário mínimo & 30 & 19 & 63,3 & 26 & 14 & 53,8 & $1,48(0,44-5,02)$ & 0,417 \\
\hline Renda per capita $<R \$ 71,50$ & 30 & 3 & 10,0 & 26 & 2 & 7,7 & $1,33(0,16-12,86)$ & 0,569 \\
\hline \multicolumn{9}{|l|}{ Condições de gestação, nascimento e saúde } \\
\hline $\begin{array}{l}\text { Início do pré-natal: } 2^{\circ} \text { trimestre da } \\
\text { gestação }\end{array}$ & 29 & 8 & 27,6 & 26 & 5 & 19,2 & $1,60(0,38-6,95)$ & 0,466 \\
\hline Número de consultas de pré-natal $<5$ & 30 & 7 & 23,3 & 26 & 10 & 38,5 & $0,49(0,13-1,81)$ & 0,219 \\
\hline Parto cesárea & 30 & 14 & 46,7 & 26 & 10 & 38,5 & $1,40(0,42-4,73)$ & 0,536 \\
\hline Peso de nascimento $\leq 3000 \mathrm{~g}$ & 30 & 12 & 40,0 & 26 & 11 & 42,3 & $0,91(0,27-3,06)$ & 0,861 \\
\hline Tempo de gestação <37 semanas & 30 & 2 & 6,7 & 26 & 5 & 19,2 & $0,30(0,04-2,05)$ & 0,155 \\
\hline $\begin{array}{l}\text { Número de irmãos que moram com a } \\
\text { criança } \geq 2\end{array}$ & 20 & 7 & 35,0 & 18 & 8 & 44,4 & $0,67(0,15-3,05)$ & 0,552 \\
\hline Não iniciou leite materno na sala de parto & 29 & 11 & 37,9 & 26 & 15 & 57,7 & $0,45(0,13-1,52)$ & 0,142 \\
\hline Início do uso da chupeta $\leq 30$ dias & 24 & 21 & 87,5 & 18 & 8 & 44,4 & $8,75(1,56-55,70)$ & 0,005 \\
\hline Criança já chupou o dedo & 30 & 1 & 3,3 & 26 & 3 & 11,5 & $0,26(0,01-3,25)$ & 0,253 \\
\hline Criança já foi internada & 30 & 9 & 30,0 & 26 & 7 & 26,9 & $1,16(0,31-4,42)$ & 0,799 \\
\hline Anemia $<11,0 \mathrm{~g} / \mathrm{dL}$ & 30 & 20 & 66,7 & 26 & 13 & 50,0 & $2,00(0,59-6,89)$ & 0,206 \\
\hline
\end{tabular}


Tabela 2 - Prevalência de fatores de risco, Odds Ratios (OR) brutos e ajustados e respectivos intervalos de confiança de 95\% (IC95\%) para as variáveis relacionadas ao período insuficiente de desmame de lactentes de nove a 18 meses

\begin{tabular}{|c|c|c|c|c|c|c|c|c|}
\hline \multirow{3}{*}{ Variáveis } & \multicolumn{7}{|c|}{ Período insuficiente de desmame } & \multirow{3}{*}{$\begin{array}{l}\text { Valor } \\
\text { de } p\end{array}$} \\
\hline & \multicolumn{3}{|c|}{$\leq 2$ meses } & \multicolumn{3}{|c|}{$>2$ meses } & \multirow{2}{*}{$\begin{array}{c}\text { OR Bruto } \\
\text { (IC 95\%) }\end{array}$} & \\
\hline & $\mathrm{n}_{\text {total }}$ & $\mathbf{n}$ & $\%$ & $\mathrm{n}_{\text {total }}$ & $\mathbf{n}$ & $\%$ & & \\
\hline \multicolumn{9}{|l|}{ Variáveis sociodemográficas e econômicas } \\
\hline Sexo masculino & 36 & 16 & 44,4 & 20 & 12 & 60 & $0,53(0,15-1,87)$ & 0,265 \\
\hline Creche pública & 36 & 16 & 44,4 & 20 & 4 & 20 & $3,20(0,77-14,29)$ & 0,067 \\
\hline Tempo de creche $<7$ meses (mediana) & 36 & 14 & 38,9 & 20 & 5 & 25 & $1,91(0,49-7,78)$ & 0,292 \\
\hline Mãe mora sem companheiro & 36 & 9 & 25 & 20 & 4 & 20 & $1,33(0,30-6,29)$ & 0,469 \\
\hline Filho caçula & 36 & 25 & 69,4 & 20 & 13 & 65 & $1,22(0,32-4,59)$ & 0,733 \\
\hline Primeiro filho & 36 & 11 & 30,6 & 20 & 7 & 35 & $0,82(0,22-3,08)$ & 0,733 \\
\hline Idade $>25$ anos & 36 & 21 & 58,3 & 20 & 13 & 65 & $0,75(0,24-2,34)$ & 0,625 \\
\hline Moradia alugada ou cedida (não própria) & 36 & 29 & 80,6 & 20 & 15 & 75 & $1,38(0,31-6,14)$ & 0,435 \\
\hline Uso de banheiro coletivo & 36 & 9 & 25 & 20 & 2 & 10 & $3,00(0,50-23,17)$ & 0,158 \\
\hline Coleta de lixo $\leq 3$ vezes por semana & 36 & 18 & 50 & 20 & 13 & 65 & $0,54(0,15-1,92)$ & 0,279 \\
\hline Mãe desempregada & 36 & 9 & 25 & 20 & 4 & 20 & $1,33(0,30-6,29)$ & 0,469 \\
\hline Emprego da mãe não qualificado & 32 & 15 & 46,9 & 18 & 8 & 44,4 & $1,10(0,29-4,17)$ & 0,868 \\
\hline Chefe da família desempregado & 26 & 1 & 3,8 & 17 & 1 & 5,9 & $0,64(0,02-26,04)$ & 0,640 \\
\hline Emprego do chefe não qualificado & 25 & 6 & 24 & 16 & 2 & 12,5 & $2,21(0,31-19,06)$ & 0,314 \\
\hline Renda familiar $\leq 3$ salários mínimos & 36 & 22 & 61,1 & 20 & 9 & 45 & $1,92(0,55-6,83)$ & 0,245 \\
\hline Renda per capita < 1 salário mínimo & 36 & 24 & 66,7 & 20 & 9 & 45 & $2,44(0,69-8,87)$ & 0,114 \\
\hline \multicolumn{9}{|l|}{ Condições de gestação, nascimento e saúde } \\
\hline Início pré-natal: $2^{\circ}$ trimestre da gestação & 35 & 11 & 31,4 & 20 & 2 & 10 & $4,13(0,70-31,29)$ & 0,067 \\
\hline Número de consultas de pré-natal $<5$ & 36 & 10 & 27,8 & 20 & 7 & 35 & $0,71(0,19-2,73)$ & 0,573 \\
\hline Parto cesárea & 36 & 15 & 41,7 & 20 & 9 & 45 & $0,87(0,25-3,06)$ & 0,809 \\
\hline Peso de nascimento $\leq 3000 \mathrm{~g}$ & 36 & 15 & 41,7 & 20 & 8 & 40 & $1,07(0,30-3,82)$ & 0,903 \\
\hline Tempo de gestação <37 semanas & 36 & 5 & 13,9 & 20 & 2 & 10 & $1,45(0,21-12,35)$ & 0,512 \\
\hline $\begin{array}{l}\text { Número de irmãos que moram com a } \\
\text { criança } \geq 2\end{array}$ & 25 & 12 & 48 & 13 & 3 & 23,1 & $3,08(0,56-18,80)$ & 0,136 \\
\hline Não iniciou leite materno na sala de parto & 36 & 19 & 52,8 & 19 & 7 & 36,8 & $1,92(0,53-7,09)$ & 0,260 \\
\hline Início do uso da chupeta $\leq 30$ dias & 30 & 22 & 73,3 & 12 & 7 & 58,3 & $1,96(0,39-10,13)$ & 0,342 \\
\hline Criança já chupou o dedo & 36 & 3 & 8,3 & 20 & 1 & 5 & $1,73(0,14-47,18)$ & 0,549 \\
\hline Criança já foi internada & 36 & 11 & 30,6 & 20 & 5 & 25 & $1,32(0,33-5,50)$ & 0,659 \\
\hline Anemia $<11,0 \mathrm{~g} / \mathrm{dL}$ & 36 & 24 & 66,7 & 20 & 9 & 45 & $2,44(0,69-8,87)$ & 0,114 \\
\hline
\end{tabular}


amostra de crianças com tempo de aleitamento materno exclusivo menor que dois meses, observou-se que $87,5 \%$ das crianças já tinham iniciado o uso da chupeta antes dos 30 dias de vida, o que pode dificultar o estabelecimento da lactação. Já no grupo de crianças com tempo de aleitamento materno exclusivo maior que dois meses, observou-se que $55,6 \%$ destas crianças iniciaram o uso da chupeta somente após 30 dias de vida.

A análise das razões de chance de riscos evidenciou que o início do processo de desmame está relacionado a: uso precoce de chupeta, como citado anteriormente (OR 8,75; IC95\% 1,56-55,70), famílias que apresentam renda menor ou igual a três salários mínimos (OR 3,73; IC95\% 1,07-13,50), criança matriculada há menos de sete meses na creche (OR 3,67; IC95\% 0,94-15,02); idade materna maior de 25 anos (OR 3,20 ; IC95\% 1,05-9,81) e mãe que mora sem companheiro (OR 3,83; IC95\% 0,79-20,91).

O modelo conceitual hierárquico de regressão logística para desmame precoce demonstrou que renda familiar menor ou igual a três salários-mínimos $(p=0,020)$, idade materna maior de 25 anos $(p=0,016)$, mãe que vive sem companheiro $(p=0,024)$ e início do uso de chupeta com menos de 30 dias de vida $(p=0,005)$ são fatores de risco independentes para o desmame precoce (Tabela 3).

$\mathrm{Na}$ construção do modelo conceitual hierárquico (Tabela 3), o mais alto nível hierárquico foi composto por variáveis socioeconômicas que indicam os efeitos dos processos estruturais da sociedade. Neste grupo, a variável "renda familiar $\leq 3$ salários mínimos" foi a que se associou com mais frequência ao desmame precoce (OR 3,73; $p=0,02)$.
O segundo nível hierárquico foi composto por variáveis indicadoras da família da criança e identificou como fatores de risco independentes para o desmame precoce: idade materna maior de 25 anos (OR 4,91; $p=0,016)$ e a mãe morar sem companheiro (OR 6,41; $p=0,024)$.

O terceiro nível hierárquico foi composto por variáveis indicadoras dos processos individuais da criança (fatores biológicos, condições de gestação, nascimento e saúde). Nesse nível, a variável introdução precoce da chupeta (antes dos 30 dias de vida) foi a única que apresentou associação estatisticamente significativa com desmame precoce (OR 8,75; $p=0,005)$.

A análise das razões de chance de riscos para o período insuficiente de desmame, utilizando o mesmo critério de $p<0,10$, indicou como possíveis riscos a criança ser matriculada em creche pública (OR 3,20; IC95\% 0,77-14,29) e a mãe ter iniciado o pré-natal somente no segundo trimestre da gestação (OR 4,13; IC95\% 0,70-31,29). No entanto, quando ajustado o modelo de regressão logística para controle de covariáveis ou variáveis de confundimento, não se obteve significância estatística.

\section{Discussão}

A importância do aleitamento materno é amplamente discutida pela comunidade científica, especialmente quando se trata da população menos favorecida, já que a amamentação é fator de proteção contra doenças diarreicas, infecciosas e do trato respiratório ${ }^{(13,19)}$, e, principalmente, de crianças assistidas em creches ou em outras comunidades fechadas, nas quais é mais fácil a transmissão de patógenos ${ }^{(20)}$.

Tabela 3 - Prevalência de fatores de risco, Odds Ratio (OR) brutos e ajustados e respectivos intervalos de confiança de 95\% (IC95\%) para as variáveis relacionadas ao desmame precoce de lactentes de nove a 18 meses

\begin{tabular}{|c|c|c|c|c|}
\hline \multirow{2}{*}{ Fatores de risco } & OR bruta & OR ajustada & \multirow{2}{*}{ Valor de $p$} & \multirow{2}{*}{ Modelo } \\
\hline & (IC95\%) & (IC95\%) & & \\
\hline \multicolumn{5}{|l|}{ Renda da família } \\
\hline$\leq 3$ salários mínimos & $3,73(1,07-13,50)$ & $3,73(1,23-11,34)$ & 0,02 & 1 \\
\hline \multicolumn{5}{|l|}{ Idade materna } \\
\hline$>25$ anos & $3,20(1,05-9,81)$ & $4,91(1,35-17,95)$ & 0,016 & 2 \\
\hline \multicolumn{5}{|l|}{ Ausência paterna } \\
\hline Mãe mora sem companheiro & $3,83(0,79-20,91)$ & $6,42(1,28-32,20)$ & 0,024 & 2 \\
\hline \multicolumn{5}{|l|}{ Uso de chupeta } \\
\hline Início do uso $\leq 30$ dias & $8,75(1,56-55,70)$ & $8,75(1,90-40,23)$ & 0,005 & 3 \\
\hline
\end{tabular}


A duração mediana do aleitamento materno de quatro meses foi menor do que os 5,6 meses observados no estudo de Spinelli com crianças menores de 18 meses frequentadoras de 12 creches do município de São Paulo em $2000^{(20)}$. Este período também é bem inferior aos 9,8 meses observados nas capitais brasileiras na última pesquisa, em $1999^{(21)}$. No entanto, o tempo de aleitamento materno exclusivo de dois meses na amostra é muito próxima das medianas observadas em estudos nacionais mais recentes de 1,$5 ; 2,3$ e 2,7 meses $^{(21-24)}$. Porém, mesmo a mediana sendo maior do que os 23,4 dias observados no país em $1999^{(21)}$, está distante da recomendação de aleitamento exclusivo do Ministério da Saúde.

Dos motivos alegados neste estudo para a introdução dos alimentos no primeiro ano de vida, ressalta-se que a principal causa foi a orientação médica. Sabe-se que a falta de preparo dos profissionais de saúde no aconselhamento sobre aleitamento materno pode determinar menor duração desta prática essencial, o que confirma a necessidade de formação continuada e treinamento atualizado desses profissionais por meio de políticas de saúde de incentivo da amamentação, principalmente a exclusiva ${ }^{(10,11,25)}$. Portanto, os fatores relacionados ao trabalho materno não se apresentaram como causa principal para a introdução de alimentos, fato também observado em outro estudo com crianças em creches em São Paulo ${ }^{(22)}$. Isso demonstra que a creche tem importante papel no incentivo à manutenção do aleitamento, permitindo que a mãe possa comparecer para amamentar no horário disponível, oferecendo local tranquilo para o ato e incentivando-a a não deixar de amamentar a criança também em sua casa.

O fato de ser o chá o primeiro alimento introduzido nesta população de crianças demonstra o hábito cultural decorrente da crença, já discutida na literatura, de que a criança tem sede e que o chá tem função terapêutica de acalmar e aliviar as dores de cólica ${ }^{(19,22)}$.

$\mathrm{O}$ uso da chupeta, aqui identificado como fator de risco para desmame precoce, foi também apontado por diversos estudos nacionais e internacionais ${ }^{(13,26-29)}$; porém, sendo um estudo transversal, não foi possível identificar se o uso de chupeta é causa de desmame precoce devido à diferença de sucção do bico do seio e do bico artificial, denominada "confusão de bicos" ${ }^{28,29)}$, que pode levar a criança a mamar com menor frequência, ou se o uso de chupeta é um marcador de dificuldade da amamentação ${ }^{(19,26,27,30)}$. É possível, contudo, apontar que o uso da chupeta deva ser contraindicado nos primeiros meses de aleitamento materno para que o desmame precoce não seja induzido.
Renda familiar menor ou igual a três salários mínimos representa risco três vezes superior do que a presença de renda maior para o desmame precoce. Trata-se de famílias em situação socioeconômica desfavorável, compostas em sua maioria por quatro membros $(69,6 \%)$, o que justifica a necessidade de a mãe ter um emprego para contribuir com a renda e a sobrevivência da família ${ }^{(11)}$ e o que não permite o aleitamento materno exclusivo pelo período recomendado.

A idade da mãe maior de 25 anos, identificada neste estudo como fator de risco para a redução do aleitamento exclusivo, difere de estudos mais recentes que demonstram a tendência de mães de maior idade amamentarem por um período mais longo do que as mais jovens, o que indicaria que as mais velhas tem mais experiência, provavelmente por terem outros filhos ou história pregressa de sucesso em aleitamento materno, como também maior maturidade em relação aos cuidados com a alimentação de seus filhos ${ }^{(10,11,31)}$.

A mãe que vive sem companheiro apresenta risco seis vezes maior de amamentar exclusivamente por menos tempo, o que pode ser explicado pela falta de apoio recebido por esta mãe para amamentar ${ }^{(19)}$. O companheiro, mesmo não sendo o pai da criança, é muito importante para o incentivo e acolhimento na dinâmica do aleitamento materno ${ }^{(10,11)}$. Em estudo realizado por Tavares $e t a^{(32)}$, foi identificado que a presença do pai como companheiro é fator de proteção para aleitamento materno exclusivo.

A partir da análise bivariada, apesar da identificação de possíveis riscos para período insuficiente de desmame, como o fato de a criança frequentar creche pública e a mãe ter iniciado pré-natal no segundo trimestre da gestação, ao se ajustar modelo de regressão logística para controle de covariáveis ou variáveis de confundimento, não se obteve significância estatística. Desta forma, essas variáveis apenas indicam possíveis riscos para um período mais curto de desmame.

Durante a coleta de dados nas instituições estudadas, foi possível observar que as creches públicas, em sua maioria, não têm estrutura suficiente para incentivar a amamentação, como uma sala disponível para a mãe amamentar, e não estão atualizadas com as novas recomendações de aleitamento e alimentação complementar e, por isso, não incentivam a continuação do aleitamento com adequada introdução dos novos alimentos. Também é possível levantar a hipótese de que as mães iniciem o processo de introdução da alimentação complementar para preparar a criança para o ingresso na instituição ${ }^{(18)}$ por desejo próprio ou por solicitação da própria creche para facilitar sua adaptação. Desta forma, o aleitamento materno deixa de ser exclusivo ou é inter- 
rompido, permitindo que a introdução de alimentos ocorra inadequadamente.

A mãe que inicia o pré-natal somente no segundo trimestre gestacional é exposta a um período menor de orientação sobre o aleitamento materno e de como se preparar para essa prática tão importante para sua saúde e a de seu filho ${ }^{(10)}$. Em estudo realizado por Escobar $e t$ al $^{(33)}$, foi encontrada associação positiva entre receber informação sobre a importância do aleitamento materno e maior duração da amamentação, demonstrando que a orientação durante o período pré-natal é valiosa na determinação do tempo de aleitamento.

Foi possível também identificar, na análise bivariada, que a criança que entra na instituição com menos de sete meses de idade apresenta possível risco quatro vezes maior de desmame precoce; fato que pode indicar a preocupação da mãe em introduzir os novos alimentos em casa para preparar a criança para o ingresso na creche, algo que deve ser desestimulado pela própria instituição.

Diante dos dados observados, é possível concluir que características importantes relacionadas à família e à instituição creche são fatores de risco para o desmame de crian-

\section{Referências bibliográficas}

1. Monte CM, Giugliani ER. Recommendations for the complementary feeding of the breastfed child. J Pediatr (Rio J) 2004;80 (Suppl 5):S131-41.

2. Palma D. Alimentação da criança: o desmame: quando, como e por quê? In: Cardoso AL, Lopes LA, Taddei JA, editores. Tópicos atuais em nutrição pediátrica da série Atualizações Pediátricas. São Paulo: Atheneu; 2004. p. 1-10.

3. Dewey KG, Brown $\mathrm{KH}$. Update on technical issues concerning complementary feeding of young children in developing countries and implications for intervention programs. Food Nutr Bull 2003;24:5-28.

4. Weaver L, Michaelsen KF. A good start in life: breast is best, but complementary foods should not be worse. Nutrition 2001;17:481-3.

5. Balaban G, Silva GA. Protective effect of breastfeeding against childhood obesity. J Pediatr (Rio J) 2004;80:7-16.

6. World Health Organization. The optimal duration of exclusive breastfeeding. Note for the press n. 7. Geneva: WHO; 2001

7. Brasil. Ministério da Saúde. Secretaria de Política da Saúde. Guia alimentar para crianças menores de 2 anos. Brasília: Ministério da Saúde; 2002.

8. Marques RF, Lopez FA, Braga JA. Growth of exclusively breastfed infants in the first 6 months of life. J Pediatr (Rio J) 2004;80:99-105.

9. Borelli MB, Palma D, Bataglin T, Taddei JA. Custo da alimentação no primeiro ano de vida. Rev Nutr 2007;20:55-62.

10. Faleiros FT, Trezza EM, Carandina L. Aleitamento materno: fatores de influência na sua decisão e duração. Rev Nutr 2006;19:623-30.

11. Santos VL, Soler ZA, Azoubel R. Children in the first semester of life: focus on exclusive breastfeeding. Rev Bras Saúde Matern Infant 2005;5:283-91.

12. Birch LL. Os padrões de aceitação do alimento pelas crianças. Anais Nestlé 1999;57:12-20.

13. Vieira GO, Silva LR, Vieira TO, Almeida JA, Cabral VA. Hábitos alimentares de crianças menores de 1 ano amamentadas e não-amamentadas. J Pediatr (Rio J) 2004;80:411-6. ças institucionalizadas e que as novas recomendações sobre aleitamento materno e alimentação complementar ainda não são conhecidas pela família e mesmo por profissionais de saúde. Portanto, é importante que os profissionais da área, assim como de educação infantil, conheçam esses fatores de risco para que possam auxiliar na promoção do aleitamento materno. Dessa forma, a creche também deve ser foco de atualização por parte do poder público para que exerça seu papel de incentivo ao aleitamento materno e para a formação de hábitos alimentares saudáveis, fatores que contribuem para o crescimento e o desenvolvimento adequados de crianças, especialmente daquelas menores de dois anos ${ }^{(16)}$. O treinamento adequado com recomendações atualizadas sobre aleitamento materno e alimentação complementar para a formação de educadores, principais responsáveis pelo cuidado da criança matriculada em creche, constitui uma ação de grande relevância para a promoção de hábitos alimentares saudáveis da população infantil contemporânea e para a disseminação das recomendações sobre alimentação no primeiro ano de vida em suas famílias.
14. INEP. Instituto Nacional de Pesquisas Educacionais. Censo Escolar 2003. Brasília: Diário Oficial da União n 167, Seção 1, 29 de agosto de 2003.

15. Bhering E, De Nez TB. Envolvimento de pais em creche: possibilidades e dificuldades de parceria. Psic Teor e Pesq 2002;18:63-73.

16. Bógus CM, Nogueira-Martins MC, Moraes DE, Taddei JA. How mothers and educators perceive the care provided by daycare centers. Rev Nutr 2007;20:499-514.

17. Beghin I. Selecting specific nutrition intervention for incorporation into rural development projects. Philipp J Nutr 1983;36:106-14.

18. Victora CG, Huttly SR, Fuchs SC, Olinto MT. The role of conceptual frameworks in epidemiological analysis: a hierarchical approach. Int J Epidemiol 1997;26:224-7.

19. Mascarenhas ML, Albernaz EP, da Silva MB, da Silveira RB. Prevalence of exclusive breastfeeding and its determiners in the first 3 months of life in the South of Brazil. J Pediatr (Rio J) 2006;82:289-94.

20. Spinelli MGN, Sesoko EH, Souza JMP, Souza SB. A situação de aleitamento materno de crianças atendidas em creches da Secretaria da Assistência Social do município de São Paulo - região Freguesia do Ó. Rev Bras Saúde Matern Infant 2002;2:23-8.

21. Brasil. Ministério da Saúde. Pesquisa de prevalência do aleitamento materno nas capitais e no Distrito Federal. Brasília: Ministério da Saúde; 2001.

22. Silveira FJ, Lamounier JA. Prevalência do aleitamento materno e práticas de alimentação complementar em crianças com até 24 meses de idade na região do Alto Jequitinhona, Minas Gerais. Rev Nutr 2004;17:437-47.

23. Camilo DF, Carvalho RV, Oliveira EF, Moura EC. Breastfeeding prevalence among children less than two years old immunized in primary health care school services. Rev Nutr 2004;17:29-36.

24. Nejar FF, Segall-Corrêa AM, Rea MF, Vianna RP, Panigassi G. Breastfeeding patterns and energy adequacy. Cad Saude Publica 2004;20:64-71. 
25. Santiago LB, Bettiol H, Barbieri MA, Guttierrez MR, Del Ciampo LA. Promotion of breastfeeding: the importance of pediatricians with specific training. J Pediatr (Rio J) 2003;79:504-12.

26. Kramer MS, Barr RG, Dagenais S, Yang H, Jones P, Ciofani L et al. Pacifier use, early weaning, and cry/fuss behavior. JAMA 2001;286:322-6.

27. Cotrim LC, Venancio SI, Escuder MM. Pacifier use and breast-feeding in children under four months old in the State of São Paulo. Rev Bras Saúde Matern Infant 2002;2:245-52.

28. Howard CR, Howard FM, Lanphear B, Eberly S, deBlieck EA, Oakes D et al. Randomized clinical trial of pacifier use and bottle-feeding or cupfeeding and their effect on breastfeeding. Pediatrics 2003;111:511-8.

29. Soares ME, Giugliani ER, Braun ML, Salgado AC, Oliveira AP, Aguiar PR. Pacifier use and its relationship with early weaning in infants born at a Child-
Friendly Hospital. J Pediatr (Rio J) 2003;79:309-16.

30. Victora CG, Behague DP, Barros FC, Olinto MT, Weiderpass E. Pacifier use and short breastfeeding duration: cause, consequence, or coincidence? Pediatrics 1997;99:445-53.

31. Lima TM, Osório MM. Breast-feeding: profile and associated factors in children under 25 months of age in Northeast Brazil. Rev Bras Saúde Matern Infant 2003;3:305-14.

32. Tavares EM, Ruowei LI, Grummer-Strawn L, Richardson M, Marshall R, Rêgo $\mathrm{VH}$ et al. Opinions and practices of clinicians associated with continuation of exclusive breastfeeding. Pediatrics 2004;113:e283-90.

33. Escobar AM, Ogawa AR, Hiratsuka M, Kawashita MY, Teruya PY, Grisi S et al. Breast-feeding and socioeconomic cultural status: factors that lead to early weaning. Rev Bras Saúde Matern Infant 2002;2:253-61. 